\title{
A Kinematic Model for the Design of a Bicondylar Mechanical Knee
}

\author{
Felix Russell*1, Ravi Vaidyanathan ${ }^{1}$ and Peter Ellison ${ }^{1,2,3}$
}

\begin{abstract}
In this paper we present a design methodology for a bicondylar joint that mimics many of the physical mechanisms in the human knee. We replicate the elastic ligaments and sliding and rolling joint surfaces. As a result the centre of rotation and moment arm from the quadriceps changes as a function of flexion angle in a similar way to the human knee. This leads to a larger moment arm in the centre of motion, where it is most needed for high load tasks, and a smaller moment arm at the extremes, reducing the required actuator displacement. This is anticipated to improve performance:weight ratio in legged devices for tasks such as stair accent and sit-to-stand.

In the design process ligament attachment positions, femur profile and ligament lengths were taken from cadaver studies. This information was then used as inputs to a simplified kinematic computer model in order to design a valid profile for a tibial condyle. A physical model was then tested on a custom built squatting robot. It was found that although ligament lengths deviated from the designed values the robot moment arm still matched the model to within $6.1 \%$ on average. This shows that the simplified model is an effective design tool for this type of joint. It is anticipated that this design, when employed in walking robots, prostheses or exoskeletons, will improve the high load task capability of these devices. In this paper we have outlined and validated a design method to begin to achieve this goal.
\end{abstract}

\section{INTRODUCTION}

Biomimetic robots take inspiration from structures and systems seen in nature in order to improve the performance of engineering systems. It is known that walking on flat ground is more energy efficient in humans and other legged animals than mechanical walking mechanisms [1] yet humans achieve this while still being capable of performing high load tasks such as stair ascent that require moments in excess of $77 \mathrm{Nm}$ for a $70 \mathrm{~kg}$ person [2].

In the field of robotics there is currently interest in developing walking robots that harness natural limb dynamics in order to reduce energy consumption [3][4]. This has been done by adding springs to the joints or actuators to help replicate human dynamics [5][6][7]. These springs are added to the joint in series or parallel with the actuation and the result is a reduction in energy consumption for level gait [8]. An alternative approach, advanced in this work, is to attempt a more literal form of biomimicry in order to directly translate the efficiency and mechanical advantage benefits of the human joint into a mechanical system. These advantages are described in more detail in Section I-A.

This work is supported by the UK Engineering and Physical Sciences Research Council with addition funding for travel from the UK Medical Research Council and the City and Guilds College Association.

${ }^{1}$ Dept. of Mechanical Engineering, Imperial College London, UK; ${ }^{2}$ Dept. of Clinical Medicine, University of Bergen, Norway; ${ }^{3}$ Dept. of Orthopaedic Surgery, Haukeland University Hospital, Norway. *felix.russell12@imperial.ac.uk
The joint presented here copies the key features of the human knee. Geometries and design parameters are taken from the literature where possible although some simplifications are made to make manufacturing feasible. Features of the human knee joint such as: varying moment arm, rolling condyles and elastic ligaments are incorporated. In this paper we describe the design process for such a joint that employs a kinematic model, we build a prototype and experimentally validate the model.

\section{A. The human knee}

The overall structure of the human knee is that of two condyles covered in smooth cartilage at the end of the femur and tibia that roll and slide over each other throughout joint motion. These surfaces are held together by ligaments and other soft tissues to resist movement of the joint outside of the normal range [9]. The four ligaments are the anterior cruciate ligament (ACL) and posterior cruciate ligament (PCL) - these are found in the centre of the knee (Figure 1) - and the medial and lateral collateral ligaments (MCL and LCL) which are found on the sides of the joint. These ligaments are elastic [10], ensuring that the condyles are always in contact. As a result the centre of rotation in the sagittal plane moves relative to both bones as they rotate [11]. A further consequence is that the ligaments stretch varies during joint motion [12]. It is also known that these ligaments contain mechanoreceptors that signal stretch [13], [14] and that this is used in joint control [15], [16].

To drive the joint the quadriceps and hamstrings muscle groups apply force via tendons and a patella. The patella runs against the smooth surface of the femoral condyle. This tendon driven actuation, combined with the moving centre of rotation, produces a moment arm that changes as a function of angle [11][17]. High load tasks such as stair climb and sit to stand require maximum moments at flexion angles of $60^{\circ}[2]$ and $90^{\circ}$ [18], respectively. Conversely at the extremes of motion low moment arms are desirable both to reduce the moment trying to hyperextend or hyperflex the joint and to reduce the total linear distance the muscle must move. Reducing this distance in a robotic joint will lead to a smaller minimum stroke length and, therefore, actuator size. Thus it is anticipated that legged devices that replicate the human moment arm curve will have an advantage over constant moment arm joints in terms of force:weight as well as easier control at the ends of the range of motion.

\section{B. Aims of this work}

Condylar mechanical knees are already commonly found in the internal prostheses used in knee replacement surgery 


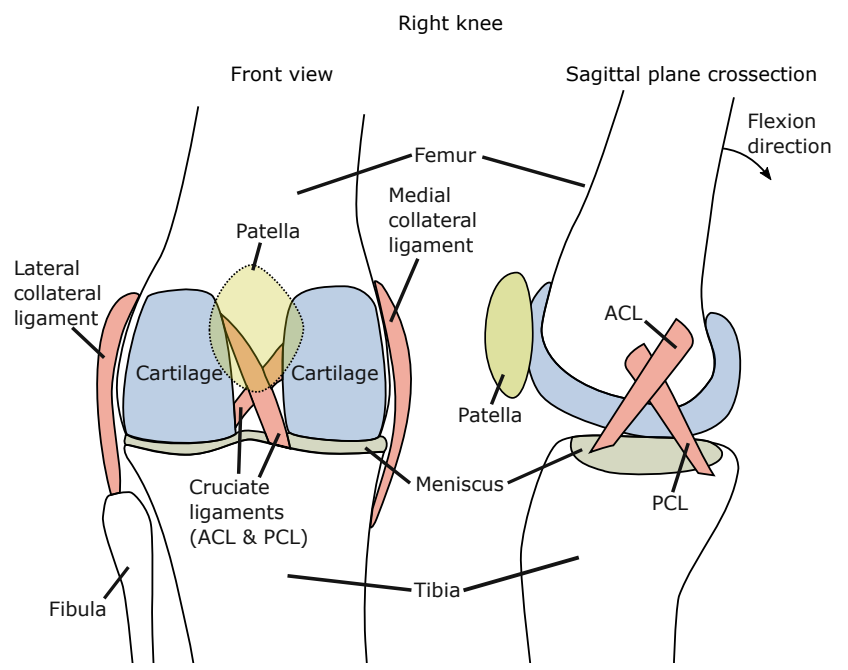

Fig. 1. Structure of the human knee joint

[19] but the authors know of no commercially available prosthetic knee for transfemoral amputees that employ this mechanism. Prosthetic knees with moving centres of rotation produced using rigid four bar mechanisms have been around for over 30 years [20] and there have been attempts to replicate the human knee moment arm curve using these mechanisms [21]. However, none of those designs have replicated the sliding, rolling and compliant sensing elements of the human knee to investigate the additional improvements to efficiency and control they may provide. Etoundi et al [22] [23] have done some initial investigation into this type of joint. Their condylar knee design contains no tendons or compliance but they found it to have a larger mechanical advantage and out of plane stiffness than a pin-jointed hinge of the same volume. We extent this work with the anticipation that a closer representation of the human knee will further improve mechanical advantage for the common high load tasks that human legs perform. Specifically, we add elastic stretch sensing ligaments and a biologically derived condyle profile. Initial data from a prototype version of the joint has shown that stretch in compliant ligaments is a function of joint forces and angular velocity as well as joint position and could provide some sensory benefit [24]. The method presented here employs a computer model developed in MATLAB to generate a tibia joint surface profile given a number of inputs taken from cadaver studies. Furthermore, we report the manufacture and testing of a physical model of the joint.

\section{Design}

Our joint has the following features:

- Two bio-derived joint condyles that can slide over each other and take the compressive load required of the joint.

- Two cruciate ligament analogues that are both elastic and have stretch sensing capability.

- Attachment points for cables that simulate tendons.
- A centre of rotation that changes position as a function of the joint angle.

Together these features produce the changing moment arm discussed in Section I-A.

Perry [25] showed that during gait sagittal plane movements dominate compared to transversal and frontal knee movements. Thus for the robotic joint these extra movement directions have been omitted. Additionally the only ligaments included were the anterior and posterior cruciate ligaments since these were deemed sufficient to stabilise the simplified geometry.

\section{DeSign MODELLING}

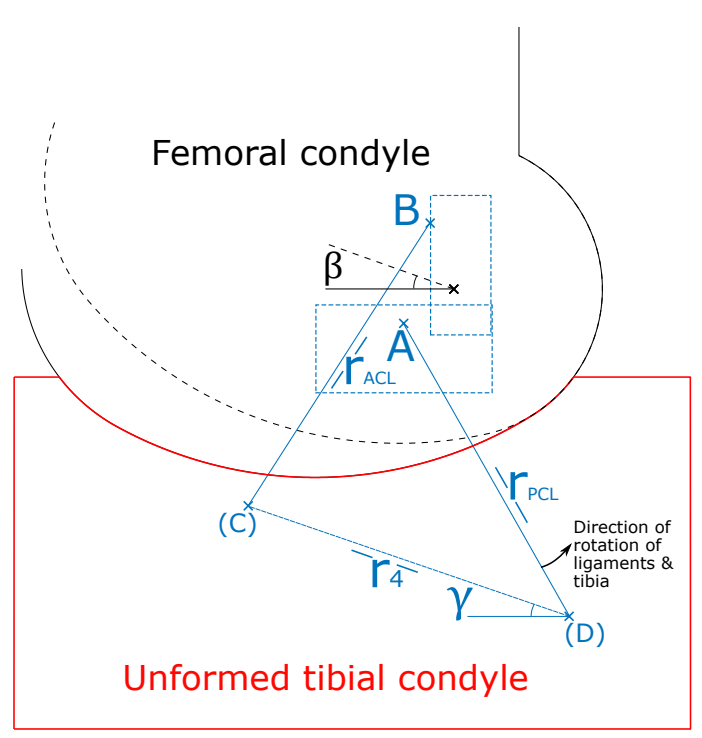

Fig. 2. The 9 inputs to the system. The locations of the femoral ligament attachment points $\mathrm{A}$ and $\mathrm{B}$ in two dimensions, the length $r_{4}$, the angle of the femoral condyle $\beta$, the angle of the ligament attachment points relative to the tibia $\gamma$ and the unstretched lengths of the ligaments $R_{A C L}^{*}$ and $R_{P C L}^{*}$. The locations A and B are constrained to within the boxes shown. These are simplified versions of the areas found by Fuss [26]

A computer model was developed as a tool which can be used to find both a profile for the tibial condyle, the path of the centre of rotation and predicted moment arm. The joint was designed to be a similar size to the human knee. However, there are no problems anticipated with scaling in order to satisfy a broad range of robotic applications. The tool has a number inputs which are both illustrated in Figure 2 and described below in more detail:

- The profile and location of the femoral condyle: The profile was derived from a sagittal plane section through a 3D scanned model of the bone performed by Isaza et al [27]. For the purposes of the model the resulting profile was approximated as three arcs. In addition an adjustment to the angle of the condyle, $\beta$, was treated as an input.

$$
0^{\circ}<\beta<20^{\circ}
$$

- The ligament lengths as a function of joint angle $r_{A C L}(\theta), r_{P C L}(\theta)$ : The ligament stretch $\left(\Delta(\theta)_{A C L}\right.$, $\Delta(\theta)_{P C L}$ ) was taken from the equally weighted average 
of the cruciate ligament bundle data from Kurosawa et al [12]. The total lengths were therefore found by adding an initial ligament lengths $\left(R_{A C L}^{*}, R_{P C L}^{*}\right)$ to these stretch measurements. This initial length was treated as an model input. The range was selected using ACL data from Cohen et al [28] and PCL values were found by taking measurements from the drawings in Fuss [26] scaled using the ACL value.

$$
\begin{aligned}
& 35.4 \mathrm{~mm}<R_{A C L}^{*}<41.4 \mathrm{~mm} \\
& 35.3 \mathrm{~mm}<R_{P C L}^{*}<41.3 \mathrm{~mm}
\end{aligned}
$$

- The location of the femoral ligament attachment points $A$ and $B$ : These were selected to be in the regions identified by Fuss [26]. The data from the Fuss paper was scaled based on the size of the posterior lobe of the femoral condyle. The regions were simplified to rectangles aligned with the system axes.

- The separation $\left(r_{4}\right)$ and angle $(\gamma)$ on the tibia of the distal ends of the ligaments $r_{4}$ : Again, these were selected to be close to the angles and lengths shown in Fuss's drawings [26].

$$
\begin{gathered}
-11^{\circ}<\gamma<29^{\circ} \\
33.7 \mathrm{~mm}<r_{4}<43.7 \mathrm{~mm}
\end{gathered}
$$

\section{A. Iterative generation of tibial condyle}

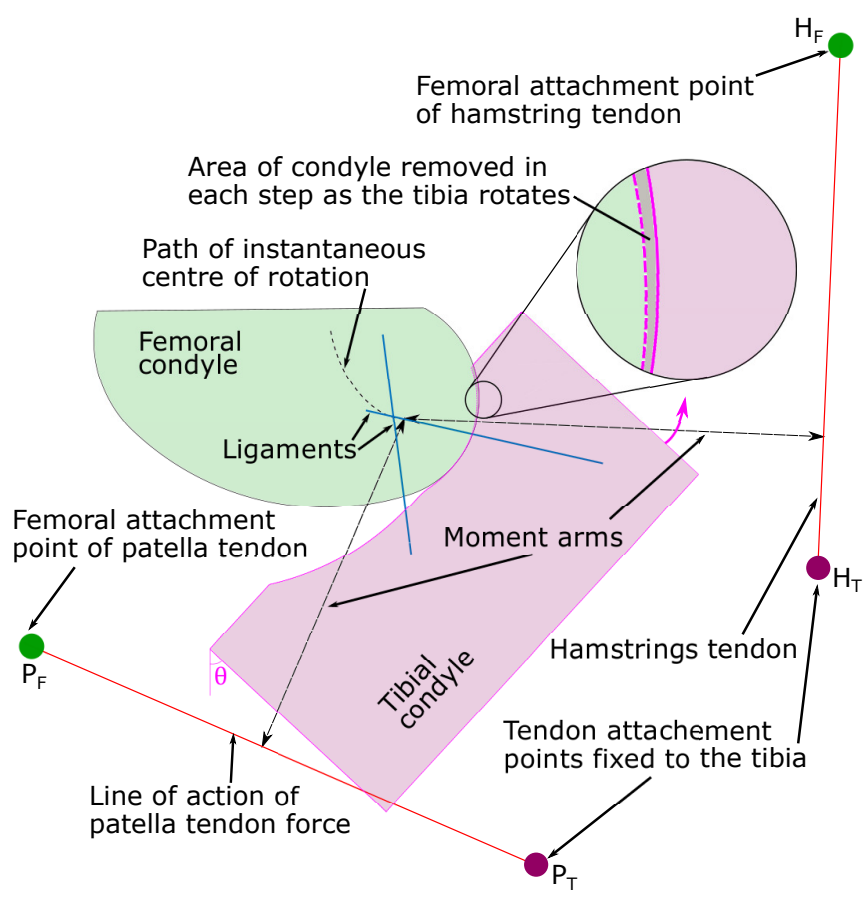

Fig. 3. Forming the tibial condyle profile

The tibia profile is formed by fixing a rectangle aligned with the system axis when $\theta=0$ to a coordinate system that moves rigidly with $r_{4}$ (Figure 2). The angle $\theta$ is increased incrementally.

In each loop of the script for $\theta_{n}=\{0,0.3,0.6 \ldots 120\}$ :

1) The angle of $r_{4}$ is updated.

$$
\overrightarrow{r_{4}}=\left|r_{4}\right| \angle\left(\gamma-\theta_{n}\right)
$$

2) The new lengths of $r_{A C L}(\theta), r_{P C L}(\theta)$ are found:

$$
\begin{aligned}
& \left|r_{A C L}(\theta)\right|=\left|R_{A C L}^{*}+\Delta(\theta)_{A C L}\right| \\
& \left|r_{P C L}(\theta)\right|=\left|R_{P C L}^{*}+\Delta(\theta)_{P C L}\right|
\end{aligned}
$$

3) The arrangement of the four bar mechanism is resolved for the new geometry. The only unknowns being the angles of $r_{A C L}$ and $r_{P C L}$

$$
\overrightarrow{r_{A B}}+\overrightarrow{r_{A C L}}+\overrightarrow{r_{4}}+\overrightarrow{r_{P C L}}=0
$$

4) The tibia is moved rigidly with $r_{4}$. Any parts of it that intersect the femoral condyle are removed (Figure 3)

5) The instantaneous centre of rotation (ICR) of the tibia was found by investigating the change in position of the points $\mathrm{C}$ and $\mathrm{D}$. The change in location of $\mathrm{C}$ and $\mathrm{D}$ is found using (7) where the subscript indicates the location of $\mathrm{C}$ and $\mathrm{D}$ at the $n^{\text {th }}$ value of $\theta$

$$
\begin{aligned}
& \overrightarrow{\Delta C}=\overrightarrow{C_{n}}-\overrightarrow{C_{n-1}} \\
& \overrightarrow{\Delta D}=\overrightarrow{D_{n}}-\overrightarrow{D_{n-1}}
\end{aligned}
$$

Then finding the perpendicular normalised vector:

$$
\begin{aligned}
& \widehat{\Delta C^{*}}=\left[\begin{array}{cc}
0 & -1 \\
1 & 0
\end{array}\right] \frac{\overrightarrow{\Delta C}}{|\Delta C|} \\
& \widehat{\Delta D^{*}}=\left[\begin{array}{cc}
0 & -1 \\
1 & 0
\end{array}\right] \frac{\overrightarrow{\Delta D}}{|\Delta D|}
\end{aligned}
$$

Then solving for the distance between $\mathrm{C}$ and the ICR $\left(d_{C}\right)$ and the distance between D and the $\operatorname{ICR}\left(d_{D}\right)$.

$$
\left|d_{D}\right| \widehat{\Delta D^{*}}-\left|d_{C}\right| \widehat{\Delta C^{*}}=C_{n}-D_{n}
$$

The location of the centroid is then found by (10).

$$
I C R=C_{n}+\left|d_{C}\right| \widehat{\Delta C^{*}}
$$

6) The length of the moment arm between the hamstring tendon and the ICR $\left(R_{h}\right)$ and the moment arm between the patella tendon and the ICR $\left(R_{p}\right)$ are then found by (11) and (12).

$$
\begin{array}{r}
\hat{n}_{p}=\frac{P_{t}-P_{f}}{\left|P_{t}-P_{f}\right|}, \quad \hat{n}_{h}=\frac{H_{t}-H_{f}}{\left|H_{t}-H_{f}\right|} \\
\left|R_{p}\right|=\left|P_{f}-I C R-\left(\left(P_{f}-I C R\right) \cdot \hat{n}_{p}\right) \hat{n}_{p}\right| \\
\left|R_{h}\right|=\left|H_{f}-I C R-\left(\left(H_{f}-I C R\right) \cdot \hat{n}_{h}\right) \hat{n}_{h}\right|
\end{array}
$$

\section{B. Selection of input values}

As there were potentially many solutions 100 random input vectors within the constraints were selected using Latin hypercube sampling. Some solutions were unsuitable because they produced condyle profiles with sharp corners, condyle contact wasn't maintained throughout motion or the location of the ligament attachment points made manufacture impossible. Of the remaining valid solutions one was selected that had both an acceptably smooth tibial condyle profile and angles $\gamma$ and $\beta$ closest to the values from Fuss [26]. The input constraints were then reduced to a small area around this point and the process was repeated and a valid solution was selected. 


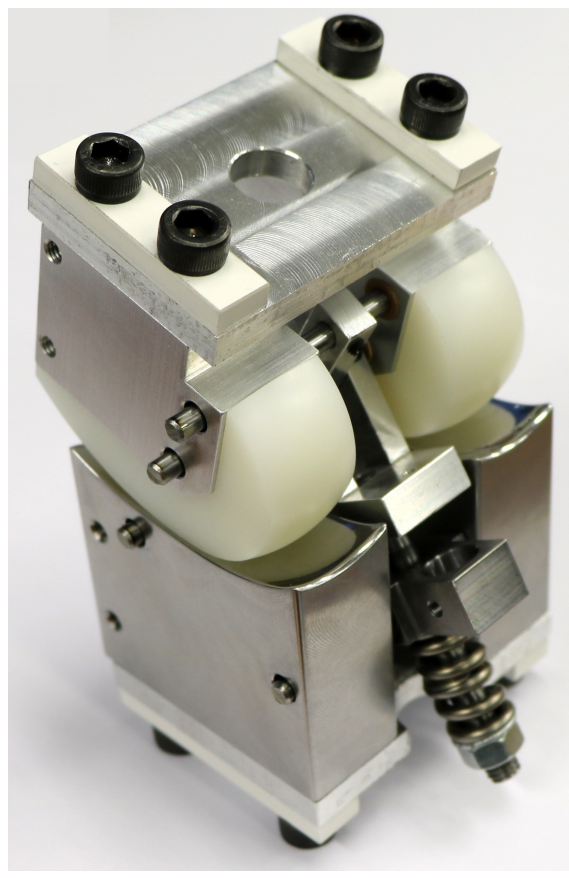

Fig. 4. Bicondylar joint

\section{EXPERIMENTAL VALIDATION}

The selected geometry was used to design and build a physical model of the joint. To maintain the two halves of the joint centred on one another a $25 \mathrm{~mm}$ radius was added to each condyle in the coronal plane. The design of the ligaments is the same as that used in Russell et al [24] with a spring for elasticity and an LVDT for stretch measurement. The free length of the ligaments (i.e. the length under no tension) can be adjusted with a nut on each one.

In addition the patella was represented as a bracket attached rigidly to the tibia. The location of this bracket and the other actuation cable attachment points was selected so that the joint moment arm fitted the human moment arm curve measured by Krevolin et al [17] with the smallest possible mean absolute difference.

\section{A. Test setup}

A test rig was built that simulated a squatting action. Active markers were placed on each limb and a rigid body tracking camera, the Optotrack Certus, was used to measure the location of the tibia relative to a coordinate system attached to the femur with an accuracy of $0.1 \mathrm{~mm}$. The vector $\left(R_{x}, R_{y}\right)$ from the location of the tibia marker $\left(x_{t}, y_{t}\right)$ to the centre of rotation was found by solving (13) for $R_{x}$ and $R_{y} \cdot d \theta$ is the change in angle achieved by the tibia in a small space of time. $d x$ and $d y$ are the change in position of the tibia over the same period.

$$
d x_{t} \mathbf{i}+d y_{t} \mathbf{j}=\left(R_{x} \mathbf{i}+R_{y} \mathbf{j}\right) \times d \theta \mathbf{k}
$$

Thus the centre of rotation $\mathbf{r}_{\text {centre }}$ could be found by (14).

$$
\mathbf{r}_{\text {centre }}=\left(R_{x}+x_{t}\right) \mathbf{i}+\left(R_{y}+y_{t}\right) \mathbf{j}
$$

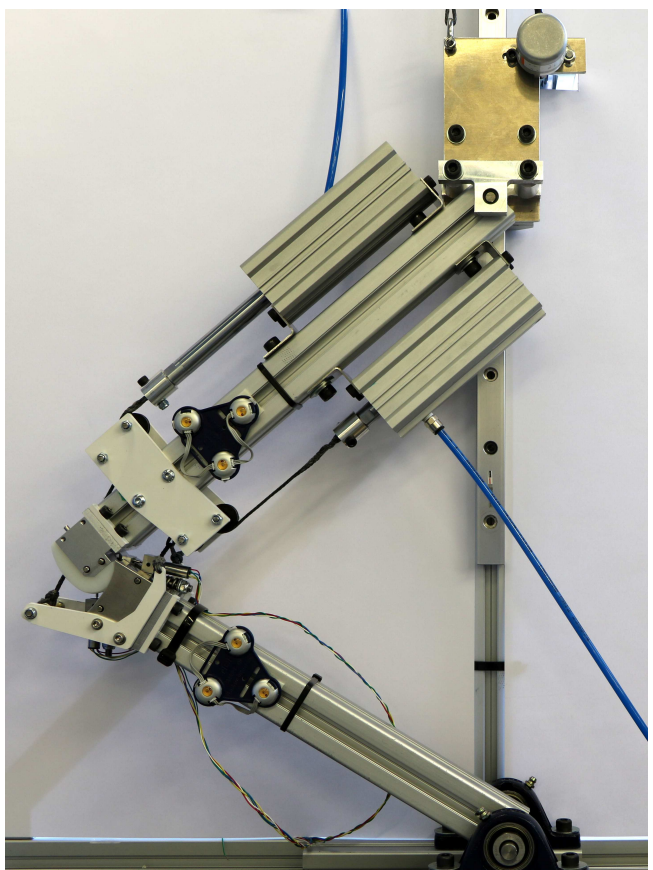

Fig. 5. Squatting test rig

The hip was fitted with a $4.5 \mathrm{~kg}$ counterweight so that the joint could be manipulated under close to no load. This was important if the kinematic model was to remain valid.

\section{B. Test procedure}

Manual manipulation of joint was performed in order to compare the parameters of the physical model with the computer model used in the design process. In order to select an appropriate free length for the ligaments the sum of absolutes differences between measured values of moment arm and ligament stretch and the equivalents in the computer model was calculated. The ligament nuts were then adjusted systematically until a compromise was found between these performance metrics. For the selected tightness 21 flexion-extension cycles were performed with a period of $5.49 s\left(\sigma^{2}=0.15\right)$. Data was collected at $200 S s^{-1}$.

\section{RESUlts}

Figure 6 shows the instantaneous centres of rotation when the joint was configured with the ligament free lengths selected in section III-B. Data has only been shown where the relative rotational velocity of the two joints was greater than $0.6 \mathrm{rad} \cdot \mathrm{s}^{-1}$. This is because as $d \theta$ in (13) approaches 0 the accuracy of the calculation decreases [29]. The mean euclidean distance between measured locations of the centres of rotation and corresponding locations from the model was $2.85 \mathrm{~mm}\left(\sigma^{2}=1.25\right)$.

The rigid attachment of the patella to the tibia meant that it's location could be found at every point in time. This, in turn, allowed an estimate of the moment arm about the centre of rotation to be calculated. The result is shown in Figure 7 alongside the moment arm estimated in the computer 
model. The mean absolute difference between the model and measured values is $2.6 \mathrm{~mm}\left(\sigma^{2}=1.3\right)$ or $6.1 \%\left(\sigma^{2}=8.3\right)$ of the model moment arm.

Figure 8 shows the measured PCL and ACL lengths alongside those from the computer model which are, in turn, derived from data from Kurosawa et al [12]. The mean absolute difference between the model and the measured values was $1.35 \mathrm{~mm}\left(\sigma^{2}=0.86\right)$ and $0.25 \mathrm{~mm}\left(\sigma^{2}=0.023\right)$ for the ACL and PCL, respectively. As maximum flexion was approached it was observed that the ACL tension reduced up to the point at which the tension in the spring was almost zero. Tightening the ACL nuts reduced this effect but also reduced the overall ACL lengths and, as a result, increased the force required to fully extend the joint.

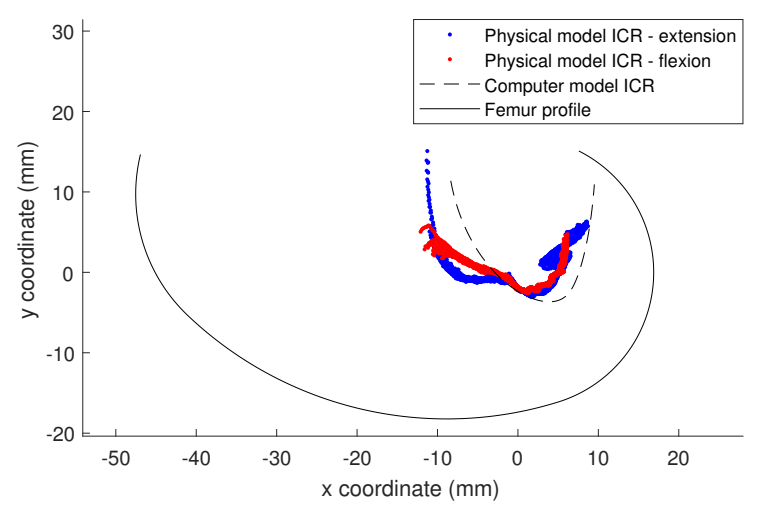

Fig. 6. Measured instantaneous centre of rotation (ICR)

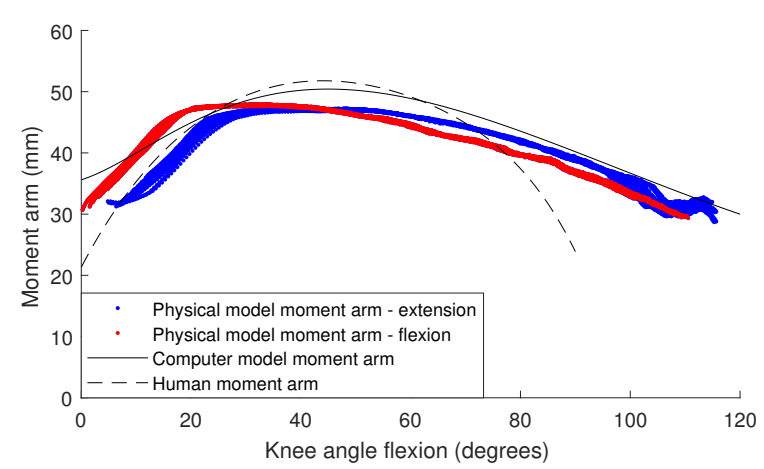

Fig. 7. Measured and model patella moment arm alongside the human patella moment arm measured by Krevolin et al [17]

\section{DISCUSSION}

Our computer model (described in Section II) allows a tibial condyle profile to be calculated with both ligament attachment points and femur profile derived from the human knee. This is performed using a simplified kinematic computer model of the joint.

\section{A. Model quality}

The computer model accounts for neither the ligament forces nor the friction between the surfaces. Indeed, although

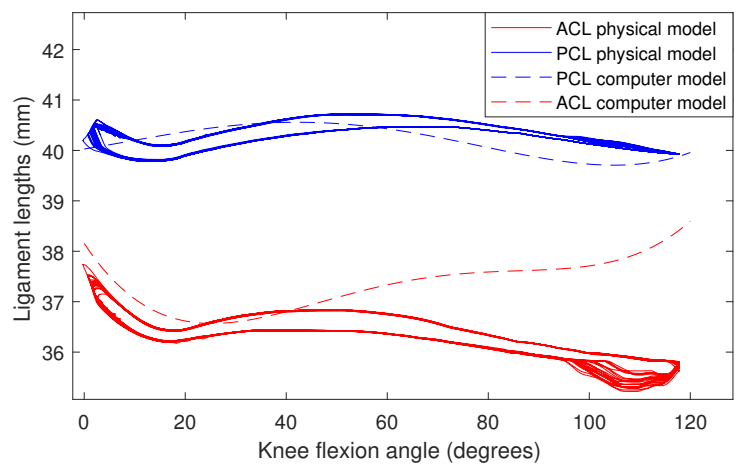

Fig. 8. Measured ACL and PCL stretch

it produces a tibia profile that allows the ligaments to stretch in the preprescribed way, it does not force them to do so. In reality the forces between the surfaces of the joint interact in a complex way with the ligament forces. However, developing, and more crucially, validating a solid body model that took these forces into account would have required an impractically long development cycle.

One outcome of these simplifications was a lower than predicted ACL length (Figure 8). Despite this the path of the rotation centres (Figure 6) follows a similar shape to the computer model, moving anteriorly as the joint is flexed as well as proximally at each extreme. There is an overall shift of the path anteriorly compared to the trajectory predicted by the computer model. Furthermore, these differences in ligament stretch and ICR had little effect on the moment arm with an average difference of $6.1 \%$ between the computer and physical models. The deviation is larger close to maximum extension but this region of motion, if anything, benefits for the reduced moment arm (see section I-A) and the fit to the human moment arm is, in fact, improved.

\section{B. Deviation in ligament stretch}

The lower than expected ACL stretch, especially near maximum flexion, suggests that the femoral condyle is shifted anteriorly in the physical model compared to the computer model. This is consistent with the anterior shift in ICR path (Figure 6). This is likely a result of the kinematic simplification of the system. However, it does not necessarily show that the resultant design is a poor representation of the human joint. The data for total ligament stretch in the computer model came from information on stretch in individual ligament bundles from Kurosawa et al [12]. An equal weight average of the stretch in the four ACL bundles was used. In flexion in particular, the difference between the amount of stretch in the different bundles in the Kurosawa data is large. Indeed, the posterior bundles do not exhibit the increases in stretch during flexion seen by the anterior bundles. As a result the range of stretch values in the data from Kurosawa et al [12] suggests that our robotic knee is in fact within the bounds of that found in humans and that the joint is a valid representation of the human mechanism. 


\section{Test procedure}

Because the computer model was strictly kinematic and did not include actuator forces it could only be validated under these same conditions. For this reason the joint was manipulated by hand. Although previous work on an earlier version of the joint suggests that these forces are likely to have an effect on the ligament stretch [24] the effect of these forces on the centre of rotation and moment arm is not known. A full investigation of how these loads move the centres of rotation and how this effects joint performance will be a matter for future work. However, the evidence presented here suggests that even when deviations in ligament stretch are large the moment arm and centre of rotation can still closely match the computer model and the human data. This supports the premise that the joint will most likely retain the mechanical advantages of the human geometry for improving high load task capability even under load.

\section{CONCLUSION}

A basic kinematic computer model has been developed in MATLAB for the design of a bicondylar joint. Specifically, the model aids with the design of a tibia profile and uses inputs taken from cadaver studies. A prototype joint has been manufactured using the computer model and this physical model has been tested in squatting under no actuator load. The most significant deviations were between the measured and model ligament stretch, in particular in the ACL. Despite this the paths of centre of rotation in both the physical and computer model matched with a mean euclidean difference of $2.85 \mathrm{~mm}$. Moreover, the joint exhibited a similar a moment arm curve to both the computer model and the human knee. This moment arm curve provides high moment capability where it is needed in the mid range of motion and reduces it where it unnecessary or dangerous at the extremes. We anticipate that walking robots, prostheses and exoskeletons will achieve an improved ability to perform both high load tasks and humanlike walking by employing our joint.

\section{REFERENCES}

[1] S. Seok, A. Wang, M. Y. Chuah, D. Otten, J. Lang, and S. Kim, "Design principles for highly efficient quadrupeds and implementation on the mit cheetah robot," in Robotics and Automation (ICRA), 2013 IEEE International Conference on. IEEE, 2013, pp. 3307-3312.

[2] P. A. Costigan, K. J. Deluzio, and U. P. Wyss, "Knee and hip kinetics during normal stair climbing," Gait \& posture, vol. 16, no. 1, pp. 31-37, 2002.

[3] P. A. Bhounsule, J. Cortell, A. Grewal, B. Hendriksen, J. D. Karssen, C. Paul, and A. Ruina, "Low-bandwidth reflex-based control for lower power walking: $65 \mathrm{~km}$ on a single battery charge," The International Journal of Robotics Research, vol. 33, no. 10, pp. 1305-1321, 2014.

[4] S. H. Collins, M. Wisse, and A. Ruina, "A three-dimensional passivedynamic walking robot with two legs and knees," The International Journal of Robotics Research, vol. 20, no. 7, pp. 607-615, 2001.

[5] T. McGeer, "Passive dynamic walking," The international journal of robotics research, vol. 9, no. 2, pp. 62-82, 1990.

[6] J. E. Pratt, "Exploiting inherent robustness and natural dynamics in the control of bipedal walking robots," DTIC Document, Tech. Rep., 2000.

[7] E. C. Martinez-Villalpando, J. Weber, G. Elliott, and H. Herr, "Design of an agonist-antagonist active knee prosthesis," in Biomedical Robotics and Biomechatronics, 2008. BioRob 2008. 2nd IEEE RAS \& EMBS International Conference on. IEEE, 2008, pp. 529-534.
[8] E. C. Martinez-Villalpando, L. Mooney, G. Elliott, and H. Herr, "Antagonistic active knee prosthesis. a metabolic cost of walking comparison with a variable-damping prosthetic knee," in Engineering in Medicine and Biology Society, EMBC, 2011 Annual International Conference of the IEEE. IEEE, 2011, pp. 8519-8522.

[9] F. G. Girgis, J. L. Marshall, and A. A. M. JEM, "The cruciate ligaments of the knee joint: Anatomical. functional and experimental analysis." Clinical orthopaedics and related research, vol. 106, pp. 216-231, 1975.

[10] R. Jones, N. Nawana, M. Pearcy, D. Learmonth, D. Bickerstaff, J. Costi, and R. Paterson, "Mechanical properties of the human anterior cruciate ligament," Clinical Biomechanics, vol. 10, no. 7, pp. 339-344, 1995.

[11] G. L. Smidt, "Biomechanical analysis of knee flexion and extension," Journal of biomechanics, vol. 6, no. 1, pp. 79-92, 1973.

[12] H. Kurosawa, K.-i. Yamakoshi, K. Yasuda, and T. Sasaki, "Simultaneous measurement of changes in length of the cruciate ligaments during knee motion." Clinical orthopaedics and related research, vol. 265, pp. 233-240, 1991.

[13] T. Hogervorst and R. A. Brand, "Current concepts reviewmechanoreceptors in joint function," J Bone Joint Surg Am, vol. 80, no. 9, pp. 1365-1378, 1998.

[14] W. Ferrell, "The adequacy of stretch receptors in the cat knee joint for signalling joint angle throughout a full range of movement." The Journal of physiology, vol. 299, p. 85, 1980.

[15] A. W. Kim, A. M. Rosen, V. A. Brander, and T. S. Buchanan, "Selective muscle activation following electrical stimulation of the collateral ligaments of the human knee joint," Archives of physical medicine and rehabilitation, vol. 76, no. 8, pp. 750-757, 1995.

[16] B. L. Riemann and S. M. Lephart, "The sensorimotor system, part i: the physiologic basis of functional joint stability," Journal of athletic training, vol. 37, no. 1, p. 71, 2002.

[17] J. L. Krevolin, M. G. Pandy, and J. C. Pearce, "Moment arm of the patellar tendon in the human knee," Journal of biomechanics, vol. 37, no. 5, pp. 785-788, 2004.

[18] S. Yoshioka, A. Nagano, D. C. Hay, and S. Fukashiro, "Biomechanical analysis of the relation between movement time and joint moment development during a sit-to-stand task," Biomedical engineering online, vol. 8, no. 1, p. 27, 2009.

[19] B. C. Carr and T. Goswami, "Knee implants-review of models and biomechanics," Materials \& Design, vol. 30, no. 2, pp. 398-413, 2009.

[20] M. P. Greene, "Four bar linkage knee analysis," Orthot Prosthet, vol. 37, no. 1, pp. 15-24, 1983.

[21] S. Pfeifer, R. Riener, and H. Vallery, "An actuated transfemoral prosthesis with optimized polycentric knee joint," in Biomedical Robotics and Biomechatronics (BioRob), 2012 4th IEEE RAS \& EMBS International Conference on. IEEE, 2012, pp. 1807-1812.

[22] A. Etoundi, S. C. Burgess, and R. Vaidyanathan, "A bio-inspired condylar hinge for robotic limbs," ASME Journal of Mechanisms and Robotics, vol. 5, no. 3, pp. 1-8, 2013.

[23] A. C. Etoundi, R. Vaidyanathan, and S. C. Burgess, "A bio-inspired condylar hinge joint for mobile robots," in Intelligent Robots and Systems (IROS), 2011 IEEE/RSJ International Conference on. IEEE, 2011, pp. 4042-4047.

[24] F. Russell, L. Gao, P. Ellison, and R. Vaidyanathan, "Challenges in using compliant ligaments for position estimation within robotic joints," in Rehabilitation Robotics (ICORR), 2017 IEEE International Conference on. IEEE, 2017.

[25] J. Perry, J. R. Davids et al., "Gait analysis: normal and pathological function," Journal of Pediatric Orthopaedics, vol. 12, no. 6, p. 815, 1992.

[26] F. K. Fuss, "Anatomy of the cruciate ligaments and their function in extension and flexion of the human knee joint," Developmental Dynamics, vol. 184, no. 2, pp. 165-176, 1989.

[27] E. Isaza, L. García, and E. Salazar, "Determination of mechanic resistance of osseous element through finite element modeling."

[28] S. B. Cohen, C. VanBeek, J. S. Starman, D. Armfield, J. J. Irrgang, and F. H. Fu, "Mri measurement of the 2 bundles of the normal anterior cruciate ligament," Orthopedics, vol. 32, no. 9, 2009.

[29] M. M. Panjabi, V. K. Goel, and S. D. Walter, "Errors in kinematic parameters of a planar joint: guidelines for optimal experimental design," Journal of biomechanics, vol. 15, no. 7, pp. 537-544, 1982. 